\title{
¿Escocia o Cataluña? La prensa española ante el referéndum escocés de 2014
}

institucional.us.es/ambitos/

Itziar Reguero Sanz

Universidad de Valladolid

itziar.reguero@uva.es

\section{Marta Requejo Fraile}

Universidad de Valladolid

marta.requejo.fraile@uva.es

\section{Ana María Velasco Molpeceres \\ Universidad de Valladolid \\ anamaria.velasco.molpeceres@uva.es}

English Version: Scotland or Catalonia? The Spanish newspapers about the Scottish referendum for 2014

\section{Resumen}

El presente artículo analiza los discursos que se esgrimieron en las principales cabeceras de la prensa española sobre el referéndum por la independencia de

Escocia. Utilizando como técnica el análisis de contenido cualitativo, el objetivo primordial de este estudio es examinar cómo fue representado el debate que generó

el 18 de septiembre, así como las comparaciones argumentales que los diarios realizaron entre la cuestión independentista escocesa y catalana. La investigación que se presenta explora un campo novedoso y abre nuevas áreas de trabajo sobre el nacionalismo, el independentismo y el papel de los medios en la conformación de

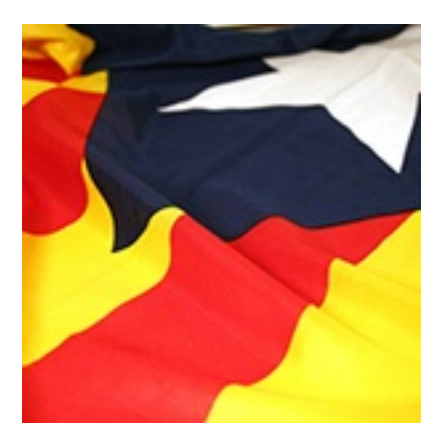
opiniones.

\section{Palabras clave \\ Cataluña, Escocia, Independentismo, Referéndum, Prensa.}

\section{Abstract}

The present paper analyses the discourses that were held in the main Spanish newspapers about the Scottish referendum for the independence. Using a qualitative content analysis technique, the aim of this paper is to examine the debate generated the 18th September and the comparisons that they made between the Scottish and Catalan independence issue. This paper explores a new and current topic in order to open new research areas of nationalism, independence and the role of the media in the public opinion.

\section{Keywords}

Catalonia, Scotland, Independence, Referendum, Press.

\section{INTRODUCCIÓN}

Esta investigación surge a raíz del impacto generado por el referéndum escocés del 18 de septiembre de 2014 en los mass media españoles. Ante el gran volumen informativo que generó dicho hito histórico, este artículo pretende abordar el papel de los principales diarios, a través del análisis del discurso que utilizaron para referirse a la votación por la independencia de Escocia. celebrar una consulta sobre el futuro político de Cataluña se realiza en un período de tiempo coincidente con el 
referéndum de Escocia, lo que hizo que entre ambos se establecieran comparaciones. Quizá la más notoria fue la aprobación simbólica de la Ley de consultas, la Ley 10/2014 (01), el 19 de septiembre de 2014, un día más tarde del esperado, pero no conseguido, 'sí' escocés.

Ante la diversidad de opiniones sobre el parecido de ambos casos, decidimos investigar los discursos que sobre esta cuestión elaboraron cuatro de las principales cabeceras de España: El País, ABC, La Vanguardia y El Mundo. A la hora de examinar la muestra de estudio, nos basamos en el supuesto de que los diarios se expresan "según un conjunto de principios, de predilecciones y de rechazos que condicionan, de cierta manera, sus comportamientos y más básicamente su percepción de la realidad” (Beneyto, 2004: 32).

\subsection{Estado de la cuestión}

Para ubicarnos en esta investigación, se han realizado rastreos bibliográficos en diferentes direcciones con el fin de encontrar una línea novedosa e inédita que nos permitiese avanzar en el campo de estudio que nos ocupa.

Si bien existen numerosas publicaciones sobre el rol de los medios escoceses en la formación de la identidad nacional (Dekavalla, 2012; Singer, 2009; Higgins, 2004; Connell, 2003; Law, 2001; Kilborn \& Meech, 1992), no lo son tantas sobre el estudio de la variable mediática en los acontecimientos del 18-S. La mayoría de los investigadores solo ha contemplado los efectos que un posible caso de independencia acarrearía en el modelo comunicativo de la región (Brown, 2014; Sutherland, 2013) o desde el punto de vista político-económico. A este respecto, solo destaca el trabajo de Forsyt (2014) sobre las consecuencias de una hipotética victoria del 'si' en Escocia desde la política conservadora británica y el de Dixon (2014), que hace hincapié en la cuestión económica si se conseguía (o no) la independencia del territorio escocés.

En el ámbito histórico, nos encontramos con varias obras. El referéndum que se celebró en el año 1997 en Escocia y Gales, durante la etapa de Tony Blair, es lo que estudia Crespo Alcázar (2007), cuya investigación nos permite contextualizar la fecha concreta que aquí se analiza. Con el mismo objetivo, se ha consultado la Tesis Doctoral de Vázquez Marruecos (1981), en la que se habla sobre el nacionalismo escocés desde una perspectiva histórica, sobre todo centrándose en el periodo comprendido desde el siglo XVII hasta el XX, además de la obra de Bergareche (2014), que estudia la estrategia de Salmond desde la perspectiva de la comunicación política, haciendo alusiones a William Wallace ('Braveheart'). A propósito de este mito de la Historia de Escocia, Perfecto García (2006) analiza los signos de la identidad escocesa a través del cine, lo cual también abarca ciertos aspectos de este análisis.

Así, cabe afirmar que no hay ninguna investigación que trate sobre cómo se abordó a través de la prensa (y mucho menos, española) el referéndum que se celebró el 18 de septiembre en Escocia, por lo que este estudio explora así un campo novedoso, de gran repercusión y actualidad que abre un nuevo área de estudio sobre el nacionalismo, el independentismo y el papel de los medios de comunicación en este proceso.

\subsection{Hipótesis y objetivos}

A la hora de analizar la prensa desde un punto de vista académico, en primer lugar, es necesario examinar cuál ha sido la interpretación y la argumentación del medio en base a lo ocurrido. Esta idea está vinculada a la Teoría del Frame Analysis o Framing: "el tema es un 'marco', un frame, que 'encuadra' la noticia dentro de áreas temáticas y guía de este modo la lectura y la interpretación del acontecimiento del que se informa”, (Boni, 2006: 112). Aunque no es fácil encontrar a autores que se refieran a la noción de frame de forma precisa (Sádaba, 2001) -hasta el punto de que algunos investigadores hablan más de la existencia de un paradigma fracturado (Entman, 1993)-, son varios los académicos que han insistido en las grandes ventajas que presenta este modelo "para explicar la función de los medios como herramientas de poder", (López \& Vicente, 2008: 8).

Según Altheide (1976), el tema y el marco de las noticias van relacionados con los 'formatos de la comunicación' que, en el caso de los medios de masas, se refiere a la selección, organización y presentación de las informaciones. Así, siguiendo a Entman (1993: 52) podemos concretar la aplicación de esta teoría en la selección de "algunos aspectos de la realidad percibida y hacerlos más prominentes en un texto comunicativo, de manera que promuevan definiciones particulares de los problemas, interpretaciones causales, evaluaciones morales y/o recomendaciones para el tratamiento del asunto descrito". De modo que, tal y como apuntan 
Tewksbury y Scheufele (2009:19), "Ios marcos informativos pueden ejercer una relativamente sustancial influencia en las creencias, actitudes y comportamientos de los ciudadanos".

Adoptando esta definición de framing y de acuerdo con Entman (1993) en su descripción de los marcos como principios de organización de la información que destacan determinados aspectos de la realidad percibida, decidimos profundizar en los marcos presentados por El País, $A B C$, El Mundo y La Vanguardia con el fin de determinar cómo fue enfocado el discurso sobre el 18-S.

Para ahondar en esta cuestión, basamos el análisis en la respuesta a la siguiente hipótesis: los periódicos identifican a Cataluña con Escocia y, a partir de ese hecho -en el que se incide en que el caso escocés es más importante para España que al contrario-, exponen sus argumentos en consonancia con su línea editorial y con un carácter muy combativo en el que las posiciones, a favor y en contra de la independencia -y a favor y en contra del paralelismo entre el caso escocés y el catalán-, están muy enconadas.

De modo que, los objetivos de los que parte esta investigación son: comparar las líneas editoriales de las cabeceras sobre el objeto de análisis, examinar qué argumentos utilizaron para referirse al referéndum escocés y a su identificación con la cuestión catalana y estudiar las comparaciones entre ambos casos.

\section{METODOLOGÍA}

Para alcanzar los objetivos planteados, la investigación ha empleado un análisis de contenido cualitativo sobre las siguientes piezas periodísticas: editoriales, artículos de opinión, noticias que abren la sección nacional, reportajes de investigación y portadas que se publicaron en las cuatro principales cabeceras españolas (El País, El Mundo, ABC y La Vanguardia)(02) entre los días 17 y 20 de septiembre de 2014 (fecha en el que se dan a conocer los resultados de la votación por la independencia de Escocia en la prensa escrita).

Han sido muchos los autores del mundo de la comunicación que han incidido en el empleo de la prensa como fuente de investigación. Dirán McClure y Patterson (1976) que este medio posee un efecto más directo en la opinión pública que la televisión debido a que ofrece más matices y elementos diferenciadores que el audiovisual. Asimismo, el periódico, "aun siendo el medio menos masivo, es, por su idiosincrasia, el que más opinión genera”, (Giménez, 2007: 18).

Así, el modelo metodológico adoptado, cuya aplicación se ha limitado al tratamiento discursivo, ha empleado técnicas de análisis de contenido para medir las interrelaciones que existían entre el referéndum de Escocia y la cuestión catalana en cada una de las páginas de los diarios de manera cualitativa. El modelo de análisis de contenido que se ha empleado en este trabajo ha quedado plasmado en la definición de Laurence Bardin (1986: 3):“El conjunto de técnicas de análisis de las comunicaciones tendentes a obtener indicadores (cuantitativos 0 no) por procedimientos sistemáticos y objetivos de descripción del contenido de los mensajes permitiendo la inferencia de conocimientos relativos a las condiciones de producción/recepción (contexto social) de estos mensajes".

Por su parte, la elección del análisis de contenido cualitativo para la investigación de este objeto de estudio ha respondido a dos cuestiones claves: tal y como explican Igartua y Humanes (2004: 75-76), porque "permite descubrir el ADN de los mensajes mediáticos [...] y examinar científicamente tanto los 'significados' como los 'significantes'". Y además, dado que este tipo de análisis se refiere a "un conjunto de técnicas sistemáticas interpretativas del sentido oculto de los textos", (Abela, 2002: 22).

Con este propósito, se diseñaron marcos de codificación para responder a cada una de las siguientes variables en las unidades de estudio:

- $\quad$ La presencia o ausencia de menciones a la cuestión catalana en el tratamiento discursivo del referéndum escocés y sus elementos de identificación y/o diferenciación.

- La presencia o ausencia de las menciones al referéndum de Escocia en el tratamiento discursivo de la cuestión catalana y sus elementos de identificación y/o diferenciación. 
- $\quad$ Los temas y personajes principales de cada una de las unidades de análisis.

- $\quad$ La caracterización de los titulares, en base a su tono y tipología.

- $\quad$ La línea editorial adoptada en la cuestión independentista y el discurso empleado en su defensa.

- Los recursos usados a favor o en contra del planteamiento editorial del diario hacia el objeto de estudio del análisis.

La respuesta a cada una de estas variables se concretó en la contestación a estas preguntas:

- ¿Cuál es la temática principal de la información?; Si es el referéndum escocés, ¿hace alguna alusión a Cataluña?, ¿Qué planteamiento utiliza?; Si es la cuestión catalana, ¿hace alguna alusión a Escocia?, ¿Qué planteamientos utiliza?

- ¿QQuiénes son los protagonistas principales? y ¿cómo son presentados?

- ¿ ¿Qué tipo de titular utiliza? y ¿por medio de qué tono se presenta?

- Autoría de la información.

- $\quad$ Lugar de emplazamiento: ¿Está enmarcada la noticia en una sección específica del tema objeto de estudio?; Si es que sí, ¿cómo titulan las páginas referidas al objeto de estudio?, ¿La información que se analiza abre la misma?

- ¿ ¿Consideraría que el periódico muestra su línea editorial en la unidad informativa?; Si es que sí: ¿Está de acuerdo el diario con el referéndum escocés? ¿Y con el que se celebraría semanas después en Cataluña?

Los resultados extraídos de cada uno de los interrogantes se han ordenado en los siguientes apartados integrando cuestiones temáticas con el planteamiento discursivo de cada una de las cabeceras. Todo ello con el propósito de determinar qué punto de vista tenía cada diario con respecto a la cuestión independentista y cómo era empleado el discurso del referéndum escocés en el tema de Cataluña.

\section{ABC: LA UNIÓN COMO GARANTÍA DE FUTURO}

$A B C$ se posicionó en contra de la separación de Escocia y el Reino Unido, así como de la celebración del propio referéndum de independencia. El diario extrapoló dicha animadversión a Cataluña, España y Europa, exponiendo las consecuencias negativas que podría acarrear para ellas un hipotético 'sí' en el territorio escocés. Así, $A B C$ aseguró que debe primar la razón frente a los sentimientos nacionalistas, mostrando una línea argumental basada en que la unión provoca la estabilidad y el progreso.

La información sobre el tema de estudio se encontró en tres secciones: Enfoque, Primer plano e Internacional dentro de estas últimas en el apartado dedicado a la cuestión escocesa: Desafío a tres siglos de Reino Unido-,donde corresponsales, columnistas y analistas reflejaron mayoritariamente la línea editorial que respalda el diario. Como muestra, la portada del día anterior al referéndum, donde se observa la bandera escocesa izada al borde de un precipicio, y un titular que reflejó la negativa del Viejo Continente: "Europa cierra filas frente al abismo escocés" (03).

La preocupación sobre las consecuencias que acarrearía la separación de un Estado para la Unión Europea fue notoria en la opinión de $A B C$. Areilza afirmó que el proceso escocés ha dejado "lecciones aprendidas" en el resto de las zonas separatistas - en las que se incluye Cataluña -, ya que quedarían claros los elevados costes que suponen los comicios y el trasfondo ético que los respalda, nada que ver con el espíritu integrador de la UE.

El diario mostró una visión crítica -y pesimista, en la mayoría de los casos- del crucial momento histórico escocés, que se identificó desde un primer momento con la situación que se estaba gestando en Cataluña. Esta idea se aprecia en numerosos titulares: "La insoportable obcecación de los nacionalismos"; "Nada volverá a ser 
igual tras la consulta"; "Temor a las secuelas del lado oscuro del sí"; "Lecciones aprendidas", y "El viaje a ninguna parte", entre otros. $A B C$ enfocó este paralelismo haciendo uso de dos de sus tesis fundamentales: Escocia y Cataluña no son lo mismo - y no se les puede tratar por igual - pero ambos han utilizado un modus operandi muy similar y, a su vez, tendrían las mismas repercusiones si triunfa un referéndum independentista.

En base a las diferencias entre dichos territorios, José María Carrascal afirmó que Escocia va a la zaga de Reino Unido, a diferencia de Cataluña, lo cual podría suponer un problema si triunfase el 'sí': "[...] el escocés es el único nacionalismo independentista que se da en la región retrasada del Estado al que pertenece. Todos los demás, el catalán, el vasco, el de la Italia Norte, surgen en las regiones ricas, que no quieren compartir su riqueza con las pobres. [...] Algo que seguro ha tenido que pesar en las reflexiones escocesas de esta noche" (04). Asimismo, Ventoso apuntó que "a diferencia de Cataluña [...] Escocia ha sido independiente durante largos periodos de su historia, desde el año 843 hasta 1707" (05).

Respecto a las similitudes de ambos nacionalismos, se señaló que tanto el escocés como el catalán han tratado de "demonizar" a los que van en contra de sus tesis e incluso, ambos líderes separatistas han respaldado sus ideas con argumentos inciertos: "Es profundamente totalitario excomulgar a los herejes unionistas, que es lo que ha hecho Salmond, y con maneras todavía más despectivas, Mas y Junqueras [...] No es en absoluto cierto que exista una mayoría independentista en Cataluña. Y tampoco les ampara la fuerza moral" (06). En base a esta idea, $A B C$ apuntó que "el nacionalismo escocés se dedicaba a falsear las consecuencias de la independencia [...] Fue la pugna entre la mentira idílica y la cruda realidad" (07). Así Rubido aseguró que actualmente vivimos "malos tiempos en los que los sentimientos se imponen a la razón [...] La mística se entremezcla con el nacionalismo, tan dado a inventar la tradición, a falsificar la Historia" (08).

Otro aspecto en el que confluyen Cataluña y Escocia, según Muns, es el de malgastar sus propios recursos en campañas que proclaman ideas separatistas: "Quebec, Escocia, el País Vasco, Cataluña y otras regiones llevan años desperdiciando recursos, energía e impuestos de la ciudadanía en campañas mediáticas dogmáticas que intentan dividir a pueblos cuya integración fortalece su capacidad de generar riqueza y empleo [...] Una constante del debate sobre el futuro de Cataluña es la obcecación de los independentistas con apelar solo a los sentimientos (de victimismo, agravios históricos) y silenciar a los técnicos que explican las realidades económicas y legales" (09).

El día que se conocieron los resultados del referéndum, de Prada clamó de una manera irónica -e inconcebible en base a sus palabras- que pese a que haya triunfado el 'no', lo sucedido había repercutido favorablemente en el independentismo de Cataluña: "Si yo fuese separatista catalán, lo ocurrido en Escocia me pondría más contento que unas castañuelas (o méscontent que un gínjol), porque sabría que en el pudridero europeo los referendos contra natura son posibles; y que, además, resultan vencedoras las pretensiones sin apoyo alguno en la naturaleza de las cosas" (10).

Este periódico también dio voz a personalidades del panorama político español como Esperanza Aguirre, que criticó el nacionalismo "exacerbado" por los efectos que generan allá donde se fraguan: "Esta votación ha dividido a la sociedad escocesa, y me atrevo a asegurar que, además, ha avivado un sentimiento de odio de los más nacionalistas hacia el resto del Reino Unido y, especialmente, hacia los ingleses [...] Fueron, precisamente, los nacionalismos exacerbados de unos y otros lo que les llevó a la carnicería más absurda y demencial de la Historia de Europa" (11).

En definitiva, y de un modo general, $A B C$ señaló que el referéndum escocés celebrado el 18-S podría haber repercutido inminentemente en el futuro del resto de países de Europa, alentando o debilitando a sus territorios con ideas separatistas: "Solo al final, e incluso pidiendo ayuda desde las embajadas británicas a miembros de Gobiernos extranjeros para que hiciesen declaraciones ad-hoc a la $B B C$, ha logrado Cameron salir del embrollo en el que él solito se metió. Y estuvo a punto de enfangarnos a todos los europeos. Como dicen los analistas más castizos '...pa' habernos matao" (12).

\section{EL MUNDO: NO A LA FRIVOLIDAD, SÍ A LA ESTABILIDAD SOCIO-ECONÓMICA}


El Mundo se sitúa completamente en contra de los movimientos independentistas, tanto escocés como catalán, centrándose su crítica sobre todo en dos aspectos: la repercusión económica y el golpe al europeísmo que supondría su triunfo.

Con el objetivo de cubrir estos dos temas, El Mundo hizo un despliegue de medios en el referéndum escocés, dado que hay un elevado número de crónicas de enviados especiales que son reseñadas como "testigos directos" en sus páginas. Es el caso de la crónica enviada por Lourdes Gómez (13), resumen de los temores del periódico hacia los partidarios del sí, pues denuncia el "tono agresivo e intimidatorio del bando contrario", lamenta que se reabran "viejas heridas" y reivindica el derecho a ser "británico y escocés".

Con el objetivo de dar una información influyente, El Mundo cuenta con numerosos análisis de expertos. Es el caso del de Manuel Medina Ortega (14), catedrático de Derecho Internacional y Relaciones Internacionales de la UCM, con el que hace un recorrido sobre la historia escocesa y plantea la cuestión de que, si bien "la celebración de un referéndum de autodeterminación en una parte del territorio del Reino Unido", no es objetable por la UE porque el "artículo 4, apartado 2 del Tratado de UE le obliga a respetar la 'identidad nacional' de los Estados Miembros", es intolerable para la Unión Europea que Escocia siga "permaneciendo como miembro de ésta después de conseguir su independencia", tal y como había propuesto el Gobierno regional escocés, pues "un Estado que se separe de su Estado matriz, al constituirse como Estado independiente, deberá solicitar el ingreso como nuevo 'Estado'” y añade que "la UE no puede favorecer el desarrollo de movimientos separatistas que debiliten la estructura de sus Estados miembros, piezas esenciales del mecanismo en que se apoya la Unión".

Llama la atención que, junto al termómetro social de las crónicas y la información de altura del análisis de los expertos, el periódico incluyó varios despieces con citas de personalidades pronunciándose sobre la independencia. Por ejemplo, el "Amo a Escocia. No necesitan a Westminster en absoluto" (15) de Morrisey o el "Escoceses, permaneced con nosotros" (16) de Bowie.

Sin embargo, es en la opinión donde la postura del periódico aparece completamente clara, tanto en sus firmas como en sus editoriales. No solo se rechaza la celebración de la consulta sino que el triunfo del 'no' escocés es visto como un fracaso, en tanto a que se celebró un referéndum a favor de la fragmentación. Así, El Mundo (17) considera que "la victoria de los unionistas deja fatalmente dividida a la sociedad escocesa y tocado políticamente al Reino Unido, uno de los países más centralistas de Europa" y critica que "el desafío que deben afrontar Cameron y los gobiernos que le sucedan", tras el auge nacionalista del "discurso neo-romántico", que no es más que "una actitud egoísta" para "apropiarse de la explotación económica de recursos".

Y, añade el periódico (18) que la situación de Europa ante estos movimientos es la de "contener la respiración", criticando que, pese a ser "casos distintos" los de Escocia y Cataluña, llama la atención "lo tarde que han reaccionado los gobiernos británicos y español frente al empuje del separatismo" pues "el soberanismo ha conseguido presentar algo tan importante como la división de un país y de una sociedad como un sencillo ejercicio democrático", cuando, bajo las supuestas "razones históricas" que arguyen los dos independentistas y su presentación como "naciones subyugadas por un Estado centralista", en realidad, hay importantes argumentos económicos. Así, en "Escocia, por ejemplo, ha calado la idea de que la gestión exclusiva de los ingresos del petróleo del Mar del Norte permitiría pagar y mejorar los servicios sociales mientras que en Cataluña ha hecho fortuna el eslogan Espanya ens roba porque la región aporta más al presupuesto del Estado de lo que recibe".

La comparación entre Cataluña y Escocia es clara, aunque no por ello se les identifica, ni es deseada por El Mundo. Aparece planteada, más bien, como algo inevitable: son dos realidades tan próximas en el tiempo, con un crecimiento del nacionalismo impulsado por la crisis económica, tan aprovechada en España por Mas la legalidad del referéndum en Escocia para apoyar el que desea en Cataluña y, a El Mundo le parece tan claro, lo egoísta, lo amenazador para Europa y la economía de sus presupuestos, que se comparan sus casos recurrentemente sin disimulo y, cuando se habla de Escocia, se lanzan órdagos contra Cataluña, más o menos discretos. Así, en todo caso (19), las consultas independentistas, independientemente de su resultado, son consideradas por el diario como generadores de "una situación perversa" pues, "aun ganando el no, el germen independentista habrá cuajado y será cuestión de tiempo que se vaya extendiendo a otras naciones de Europa”. 
Por lo que juzga que "los gobierno deberán esmerarse en encauzar el fenómeno nacionalista para evitar que degenere en una grave fragmentación política y social y en el fracaso del proyecto de la Unión Europea”.

Y este punto es crucial para El Mundo (20): la ineficacia de los líderes, tanto británicos como españoles, y la frivolidad y falta de visión de todos ellos. Lamentan que Cameron no viera que la celebración del referéndum era un problema enorme por el mero hecho de plantearse. Critican que Salmond afirme que un 'no' a la independencia aplazará la consulta por veinte años pero que en realidad ese "compromiso pueda volatilizarse con un simple cambio de Gobierno". Atacan a Rajoy y su gobierno por su tibieza, pero también a Mas por ser, en pocas palabras, un fantoche agarrado al poder, sin ninguna entidad -no deja de proponer "utopías salvíficas que prometen remedios autónomos a problemas surgidos en una economía transnacional que requiere de la cooperación multilateral"-y por completo incoherente.

Por todo esto, quizá es con Arcadi Espada, firma fija de El Mundo, con quien el periódico más coincide en el rechazo de estos movimientos independentistas. Todo el proceso es lo que Espada llama "la democracia frívola" (21) pues los escoceses "ya tuvieron su estúpido referéndum autorizado por el peor primer ministro de la Historia de Gran Bretaña y por uno de los grandes peligros europeos. Ya tuvieron el referéndum que reclamaban los votantes del Scottish National Party, un 45 por ciento en 2011", y no toda la población escocesa -consenso que al menos sería necesario para "destruir un Estado"-, por no hablar de la opinión de los "millones de británicos que están en contra de la destrucción de su Estado" y de los que nadie se ha ocupado. Además, lo perdieron, porque se han basado en que "hace 300 años fue un país independiente", obviando si "Escocia tendrá libra o euro, si estará en Europa o no, y si hasta tendrá a su reina". De manera que este referéndum, como la consulta, fija "en el imaginario escocés una idea maligna" y traerá "consecuencias funestas" pues, como dijo Gordon Brown, con él, "el país que había sido un modelo para el mundo se ha hecho más pequeño, y no más grande". Otro rasgo más de frivolidad es que "dimita Salmond y ahí quede Cameron, el estólido", como Rajoy con un Mas infantilizado y desesperado por "votar; votar como sea" (22). Así, lo mismo que se negaba a ofrecer enseñanza en castellano, para "ganarse a los casi 300.000 marroquíes de Cataluña para la causa independentista" pretende "introducir la enseñanza de la religión islámica" (23). Y es que, El Mundo lo ve claro, como Raúl del Pozo en su contraportada (24): "las independencias no se conceden, se arrancan". Con todo lo que eso supone.

\section{EL PAÍS: NO A LA SEGREGACIÓN EN EUROPA}

Durante los cuatro días en los que transcurrió el análisis, El País se mostró en contra de una posible independencia tanto de Escocia como de Cataluña; no tanto en lo que a la realización del propio referéndum se refiere, sino por las futuras repercusiones que tal separatismo acarrearía para Europa. El diario dedicó un amplio número de recursos para profundizar en dicha cuestión aportando una solución intermedia, muy acorde con su planteamiento editorial: una reforma que permita una unidad diversa y que se concreta en el modelo federal.

El País abordó esta idea, fundamentalmente, por medio de dos vías: recurriendo a análisis de expertos del mundo de la política y de la economía y realizando extensos reportajes sobre otras formas de tratar la cuestión separatista en ciertas regiones europeas. Todos ellos aparecieron recogidos en dos secciones creadas específicamente para abarcar este objeto de estudio: Referéndum en Escocia y El encaje de Cataluña en España. Sin embargo, solo se acompañó de diferentes subtítulos (como La campaña, La votación u Otros separatismos) a la primera de estas. En este sentido, cabe mencionar que el despliegue de medios empleado por el periódico para la cobertura del referéndum escocés fue mayor a la utilizada para el tratamiento de la cuestión catalana, pero siempre con una finalidad: promover un discurso a favor de la reforma de los instrumentos democráticos para adaptar las nuevas reivindicaciones a los nuevos tiempos como "proyecto político de primer orden" (25).

Así, a pesar de que autores como Lluis Bassets consideran que "ideas como éstas (refiriéndose al referéndum de Escocia y de Cataluña) se expanden a una velocidad vírica" (26), El País entiende que existen alternativas, como la creación de "un mecanismo de defensa, una señal de diálogo y una búsqueda de soluciones constructivas". No obstante, el diario es realista y considera difícil su puesta en marcha en el corto plazo: "Una 
reforma a la altura de los retos planteados no puede pactarse en una rápida negociación” (27).

Que el referéndum en Escocia es usado como punto de mira para abordar la cuestión catalana es algo que queda puesto de manifiesto en el propio nombre dado a la sección dedicada para hablar de Cataluña (El encaje de Cataluña en España), así como en las propias unidades de estudio: "Escocia no es Cataluña y sus situaciones no tienen comparación histórica ni legal, pero no pudo evitar utilizar la consulta que se celebra hoy en esa zona de Reino Unido como referencia para descalificar la que proyecta la Generalitat para el 9 de noviembre" (28). Sin embargo, en la mayor parte de las informaciones este recurso fue usado como elemento contextual, más que argumental, para exponer las tesis europeístas del diario.

Esto explica que el diario dedicara cada día una de sus páginas a abordar otros separatismos europeos como el de Flandes en Bélgica o el de la Padania en Italia, o que una vez conocido el triunfo del 'no' en Escocia, la mayoría de las informaciones y opiniones de El País se enfoquen en clave de triunfo europeísta: "La noticia es magnífica para el europeísmo. De entrada, los europeos encontrarán alivio en el alejamiento de los peores augurios: el desgarro e inestabilidad [...] el suceso de la votación [...] gratificará especialmente a los europeístas porque al Gobierno de Londres le será mucho más arduo argumentar contra los avances federales de los Veintiocho [...] Scots, be welcome home!" (29).

Asimismo, el diario constantemente invitó a la reacción frente a la parálisis y a la revitalización del espíritu europeísta sobre el que se fundó el ideal europeo hace 57 años. "Hay que repensarlo todo, pero quizá desde otros enmarques más ajustados al siglo XXI, aquellos que hoy exige una Europa cohesionada, fuerte y unida. Este es el desafío [...] Si hay un nuevo empoderamiento de lo local es porque el Estado ya no nos protege, ni social ni militarmente [...] ¿Para qué necesitamos a Madrid desde Cataluña si quienes deciden al final son Frankfort y Bruselas?" (30).

Las alusiones en tono de crítica deslegitimando la actuación de Cameron en el proceso fueron constantes: "El campo del no se despertó hace menos de dos semanas [...] hasta entonces, Londres se había limitado a bombardear a los escoceses con multitud de informes" (31); "Incluso la prensa británica reaccionó tarde ante el espectro de una Escocia independiente" (32), añadió Tubella.

En cualquier caso, clamó El País que "puede que haya llegado el momento de clarificar las cosas" y "con reforma constitucional o sin ella, habilitar una solución integradora con garantías para todas las opciones y voces. Todo, antes de que la frustración catalana se haga crónica y la burbuja de la frivolidad y el victimismo nacionalista siga campeando porque 'no les dejan votar' y lleve al país a un callejón sin salida" (33), dado que "la independencia supone invertir mucho tiempo y recursos en volver a la casilla de salida donde esperan los mismos problemas de siempre" (34).

\section{LA VANGUARDIA: ALABANZAAL REFERÉNDUM ESCOCÉS Y AL ‘ SENY BRITÁNICO’.}

Durante el periodo de estudio, La Vanguardia se mostró a favor del derecho a decidir de los pueblos, sea cual fuere su procedencia. Así, calificó el referéndum escocés como "un impecable ejercicio democrático" donde ha primado "el respeto al contrario, el diálogo y la voluntad de pacto" (35). El diario barcelonés, por proximidad y afinidad, hizo un paralelismo constante de Escocia con Cataluña, atacando el modus operandi de España ante la cuestión nacionalista y, a su vez, ensalzando la actitud conciliadora llevada a cabo en Gran Bretaña.

Las muestras de análisis se han publicado mayoritariamente en las secciones Opinión e Internacional, dentro de esta última más concretamente en el apartado Una consulta histórica. En las primeras páginas del diario, asimismo se ha reflejado que el periódico estaba de acuerdo con dichos comicios, mostrando la concordia entre los simpatizantes del separatismo y de la unión (36).

La línea argumental de La Vanguardia se ha movido en varias direcciones: en primer lugar, se trató de extrapolar lo sucedido el 18-S a un hipotético 9-N - todavía no se había celebrado la consulta -. Así, Juliana afirmó que "Escocia no es Catalunya, pero hay algo en común en su historia reciente" (37), y González señaló, citando al President de la Generalitat, "qué guapa estaría España con una falda escocesa" (38). 
A su vez, se apuntó que el resultado de los comicios en Escocia tendría repercusión directa en España, "donde se ha vivido la campaña como si fuera un asunto interno, conscientes de que, salga lo que salga, tendrá repercusiones" (39) así como en Cataluña: "[...] la próxima semana, por lo tanto, el coche de Mas se acercará al precipicio. ¿Y el de Rajoy? Dependerá de lo que ocurra en Escocia. Nosotros [los catalanes] [...] nada podemos hacer, de momento, excepto beber tila" (40). Del mismo modo, Ónega aseguró que el referéndum suscitaba gran admiración en Cataluña: “Ay quién fuera escocés!, pensarán hoy unos cuantos miles, posiblemente millones de catalanes. Escocia quizá sea mañana el escenario de una histórica decepción pero hoy es espejo, meta y envidia de cualquier buen nacionalista" (41). También se hizo referencia a las diferencias entre ambos territorios, sobre todo en lo concerniente a sus principales líderes: "Cataluña no es la historia pasada, sino la historia que queremos escribir; "Es evidente, en el plano formal, que Mariano Rajoy [...] no es David Cameron, pero tampoco Artur Mas es Alex Salmond" (42).

La alabanza a la ejecución del referéndum, tanto por parte de Escocia como de Gran Bretaña, fue la tesis principal de La Vanguardia en estos cuatro días, como muestran los siguientes titulares: "Referencia Escocia"; "Espejo escocés"; “Un impecable ejercicio democrático"; "Una fiestaza”; "La democracia como religión”, y "Una lección, una gran lección”.

En la misma línea, la admiración al talante democrático británico fue un punto unánime en los artículos publicados en La Vanguardia. Ónega alabó "[...] la forma en que se desarrolló el debate soberanista [...]: nadie se ha peleado, nadie ha insultado, nadie invocó medidas extremas y los incidentes han sido la excepción. Los rencores, que los hubo, han sido británicamente disimulados" (43). Así, se señaló que "la tradición democrática y calidad institucional del Reino Unido siguen siendo un ejemplo" (44) y que "sobre estas tablas de racionalidad se cierra un ciclo que se ha desarrollado con el toque de civismo propio de un país del norte" (45).

Por otro lado, y en relación con dicho halago al 'seny británico', se ha criticado la postura adoptada por el Gobierno español frente a la cuestión catalana. Rahola afirmó "ya sabemos, y sufrimos, que la salud democrática británica no es la española, empezando por la incapacidad patológica de España de reconocer el carácter nacional de Catalunya. [...]. De España dependerá mantenerse en la cutrería política, o alcanzar la historia" (46) y, en la misma línea, Álvaro criticó que "mientras los poderes británicos aceptan que Escocia es una nación [...] los poderes españoles no admiten que Catalunya [lo es]. En el mejor de los casos, se utiliza "nacionalidad", término exquisitamente constitucional que nadie sabe qué significa" (47).

Este autor señaló, a su vez, que "en la capital española, el referéndum escocés ha generado muchos nervios. Un espectáculo que muestra a la perfección cuál es el drama de fondo de la cultura política española, más allá y más acá de regímenes, dictaduras y restauraciones" (48). Más comedido en su argumento, Ponti afirmó que el Gobierno catalán y el español deberían ceder y escucharse mutuamente si quieren llegar a un entendimiento, ya que se plantea imposible una victoria total de ambas posturas: "Ojalá el problema político actual se pudiera analizar bajo el prisma del ganar-ganar. Pero me temo que no es así. Ni la dinámica interna de los partidos ni en las relaciones entre gobiernos impera el win-win. Sólo veo amenazas, ultimátums, comentarios despectivos y una alarmante falta de escucha. No hay colaboración sin escucha activa y empática. Tenemos dos oídos y una boca para poder escuchar el doble de lo que hablamos" (49).

Para terminar este epígrafe, se muestra un fragmento de la columnista Pilar Rahola, donde se aúnan -de un modo muy crítico y tajante- las bases en las que se centró la línea argumental de La Vanguardia sobre esta cuestión: "[...] la sociedad española, a diferencia de la británica, acepta el secuestro de las leyes del Estado, con el fin de impedir el ejercicio de la democracia", [...] Es decir, todo vale, incluso amenazar con intervenir la autonomía, es decir, practicar la violencia legal, lo cual remitiría a España a tiempos muy oscuros [...] Pueden poner muchos eufemismos y quemar toda la gramática de la Real, pero el hecho es inequívoco: votar es normal en una sociedad libre. Hoy lo demuestra Escocia. Si España no lo encuentra normal, es España la que está enferma" (50).

\section{CONCLUSIONES}

Los resultados del estudio realizado en esta investigación permiten confirmar que la cobertura del referéndum 
por la independencia de Escocia, celebrado el 18 de septiembre de 2014, tuvo un claro reflejo argumental y temático en las informaciones sobre la cuestión catalana presentadas en las páginas de los cuatro principales diarios españoles.

El posicionamiento acerca del referéndum de Escocia estuvo muy polarizado. El Mundo y $A B C$ estuvieron totalmente en contra de la votación, a diferencia de El País y La Vanguardia, quese mostraron a favor: el diario madrileño apoyó el uso de instrumentos democráticos -el referéndum en sí-, pero no se posicionó a favor de la independencia. Por otra parte, el medio barcelonés respaldó el proceso de consulta escocés en su totalidad, tanto en la forma como en el fondo.

La principal línea argumental sobre la que los diarios construyeron sus posturas fue el posible impacto del referéndum en la Unión Europea, así como en los Estados miembros. La desmembración de la UE y el miedo al 'contagio' de otras zonas con movimientos independentistas se convirtieron en las grandes preocupaciones que expusieron El Mundo, $A B C$ y El País. Este último, por temor al retroceso del europeísmo, propuso una tercera vía: el federalismo como punto medio entre el fervor nacionalista y los modelos centralistas.

La comparativa entre Escocia y Cataluña estuvo presente en los discursos de todos los medios, señalando que ambos territorios cuentan con más diferencias que similitudes. No obstante, el uso de este paralelismo se adaptó a la línea editorial de cada diario: $A B C$ señaló que no eran lo mismo, pero sí lo era el modus operandi de los territorios, así como las consecuencias negativas que la consulta acarrearía. Así, El Mundo hizo hincapié en el aspecto económico, argumentado el elevado coste del referéndum. La Vanguardia, en cambio, confrontó los dos Estados: expuso a Gran Bretaña como un modelo democrático para España, por el referéndum y por cómo se ejecutó.

En este estudio de caso se pone de manifiesto la polarización ideológica de los diarios, sobre todo por la identificación del tema con la cuestión catalana, a la cual aluden de manera velada pero evidente en las informaciones vertidas sobre Escocia. Por ello, constatamos que los principales periódicos de referencia española ejercieron como actores políticos, postergando su teórico papel como servicio público, en aras de deslegitimar o potenciar el nacionalismo catalán por asimilación al caso escocés, lo que pudo poner en peligro el conocimiento del acontecimiento en sí mismo por parte de la opinión pública española. No podemos olvidar, como afirman Giménez y Berganza (2009: 145), que los medios de comunicación "en ocasiones, se convierten en la única posibilidad de conocer lo que acontece en el mundo, y asomarse a ellos supone acercarnos a una realidad no 'fáctica' sino 'percibida', donde nuestro juicio se construye a través del conocimiento ajeno".

Sobre la base de estos hallazgos es posible verificar la hipótesis enunciada al inicio de esta investigación: los diarios objeto de estudio han realizado una identificación entre Escocia y Cataluña, construyendo las informaciones y las opiniones a través de argumentos en concordancia con la línea editorial de cada uno de los diarios. Este estudio nos ha permitido completar el vacío que existía sobre esta cuestión, y, a su vez, abrir una nueva línea de investigación, en la cual sería conveniente profundizar con el análisis de otros medios de comunicación españoles (como la televisión) o con el examen de la prensa escocesa durante la consulta catalana llevada a cabo el 9-N, trabajo paralelo que completaría el aquí expuesto.

\section{REFERENCIAS BIBLIOGRÁFICAS}

ALTHEIDE, D. (1976): Creating Reality: How TV News Distorts Events. Beverly Hills, CA: Sage.

BARDIN, L. (1986): Análisis de contenido. Madrid: Akal.

BENEYTO, J.V. (2004). Poder global y ciudadanía mundial. Madrid: Taurus.

BERGARECHE, B. (2014): “La independencia 'light' de Salmond para Escocia”. Política Exterior, n 28, pp. 118130.

BONI, F. (2006). Teorías de los medios de comunicación. Barcelona: Aldea Global. 
BROWN, M. (2014): "Everything still to fight for". Television, vol.51, n 10, pp. 16-17.

CONNELL, L. (2003): "The Scottishness of the Scottish press: 1918-39". Media, Culture \& Society, vol.25, n², pp. 187-208.

CRESPO-ALCÁZAR, A. (2007): "Els processos devolucionistes a Escócia (i al País de Gal-les)". Diàlegs: revista d'estudis polítics i socials, $\mathrm{n}^{\circ} 10$, pp. 25-47.

DEKAVALLA, M. (2012): "Evaluating newspaper performance in the public sphere: Press accounts of Westminster elections in Scotland and in England in the early post-devolution period". Journalism, vol.13, $n^{\circ} 3$, pp. 320-339.

DIXON, K. (2014): “La ambiciones del nacionalismo escocés: hacia la autodeterminación”. Le Monde diplomatique en español, $\mathrm{n}^{\circ} 227$, pp. 1-18.

ENTMAN, R.M. (1993): "Framing: Toward clarification of a fractured paradigm". Journal of Communication, vol.43, n 4 , pp. $51-58$

FORSYT, M. (2014): "Lecciones del referéndum de independencia en Escocia”. Política Exterior, n 162, pp. 26 29.

GIMÉNEZ, P. (2007): “La objetividad, ¿una cuestión deontológica?”. Revista Comunicación y Hombre, nº 4, pp. 119-129.

GIMÉNEZ, P.; BERGANZA, M.R. (2009): Género y Medios de Comunicación: Un análisis desde la Objetividad y la Teoría del Framing. Madrid: Fragua.

HIGGINS, M. (2004): "The articulation of nation and politics in the Scottish press". Journal of Language \& Politics, vol.3, n³, pp. 463-483.

HUMANES, M.L.; IGARTUA, J.J. (2004): Teoría e Investigación en Comunicación Social. Madrid: Síntesis.

KILBORN, R.; MEECH, P. (1992): "Media and identity in a stateless nation: The case of Scotland". Media, Culture \& Society, vol.14, $\mathrm{n}^{\circ} 2$, pp. 245- 259.

LAW, A. (2001): "Near and far: banal national identity and the press in Scotland". Media, Culture \& Society, vol.23, n 3, pp. 299-318.

LÓPEZ, P.; VICENTE, M.: Tendencias actuales en la investigación sobre framing: consolidación internacional y emergencia en la academia española. En AA.VV. (2008): Investigar la Comunicación. Actas del Congreso Internacional Fundacional de la Asociación Española de Investigación en Comunicación, Santiago de Compostela, 30-31 enero de 2008. España: Asociación Española de Investigación de la Comunicación.

MCCLURE, R.; PATTERSON, T. (1976): Television News and Voter Behavior. New York.

PERFECTO-GARCÍA, M.A. (2006): "A propósito de Braveheart y el mito de la Escocia Independiente: reflexiones sobre el nacionalismo escocés contemporáneo". Revista de historia actual, nº 4, pp.207-226.

PINILLA, A. (2008): La transición de papel. Madrid: Biblioteca Nueva

SÁDABA, M.T. (2001): "Origen, aplicación y límites de la 'teoría del encuadre' (framing) en comunicación”. Comunicación y Sociedad, vol.14, n² 2, pp. 143-175.

SÁNCHEZ-ARANDA, J.J.: Análisis de contenido cualitativo de medios. En: BERGANZA, M.R; RUIZ SAN ROMÁN, J.A. (2005): Investigar en comunicación. Madrid: Mc Graw-Hill, pp. 207-228.

SINGER, J. (2009): "Separate Spaces: Discourse about the 2007 Scottish Elections on a National Newspaper Web Site". International Journal of Press/Politics, vol.14, n 4, pp. 477-496. 
SUTHERLAND, E. (2013): "Media and telecommunications after Independence". Intermedia, vol.41, n 2, pp. 3436.

TEWKSBURY, D.; SCHEUFELE, D.A.: News framing theory and research. En J., BRYANT, J; \& OLIVER, M.B. (2009): Media effects: Advances in theory and research. Hillsdale, NJ: Erlbaum, pp. 17-33.

VÁZQUEZ MARRUECOS, J.L. (1981): Los nacionalismos británicos: Escocia. Tesis doctoral inédita, Universidad de Granada.

(01) Diari Oficial de la Generalitat de Catalunya: http://www.aelpa.org/actualidad/201409/LeyConsultasCatalunya.pdf [consultado: 13 de febrero de 2015].

(02) Según los datos para el periodo 2013-2014 de la Oficina de Justificación de la Difusión, se trata de los cuatro diarios más difundidos en España. Con la elección de esta muestra hemos intentado cubrir el más ampliol abanico de actitudes políticas e ideológicas del territorio nacional.

(03) Europa cierra filas ante el abismo escocés. (18 de septiembre de 2014).ABC, p. 1.

(04) Carrascal, J.M. (18 de septiembre de 2014). El dilema escocés. ABC, p. 3.

(05) Ventoso, L. (18 de septiembre de 2014). De dónde viene y a dónde va el referéndum de independencia. $A B C$, p. 22.

(06) Ventoso, L. (20 de septiembre de 2014). Suplantación. ABC, p. 14.

(07) Gran Bretaña sigue. (20 de septiembre de 2014). ABC, p. 4.

(08) Rubido, B. (20 de septiembre de 2014). Cuadro escocés. ABC, p. 4.

(09) Muns, A. (20 de septiembre de 2014). La insoportable obcecación de los nacionalismos. ABC, p. 17.

(10) De Prada, J.M. (20 de septiembre de 2014). Independencias. ABC, p. 15.

(11) Aguirre, E. (20 de septiembre de 2014). El referéndum escocés visto por una española. ABC, p. 29.

(12) Pérez Maura, R. (20 de septiembre de 2014). Quién perdió fue el Reino Unido. ABC, p. 4.

(13) Gómez, L. (2014, Septiembre, 18). La ruptura es cara y arriesgada. El Mundo, p. 21.

(14) Medina, M. (20 de septiembre de 2014). El frío fuera de la Unión Europea. El Mundo, p. 22.

(15) A favor del sí. (18 de septiembre de 2014). El Mundo, p. 22.

(16) A favor del no. (18 de septiembre de 2014). El Mundo, p. 23.

(17) La unidad política en Reino Unido consigue frenar el independentismo. (20 de septiembre de 2014). El Mundo, p. 3.

(18)Encauzar los nacionalismos para evitar la fragmentación de Europa. (18 de septiembre de 2014). El Mundo, p. 3.

(19) Íbidem.

(20) Íbidem.

(21) Espada, A. (20 de septiembre de 2014). La democracia frívola. El Mundo, p. 15.

(22) De la Serna, V. (20 de septiembre de 2014). Escocia cambia el guión de la secesión catalana. El Mundo, p. 
15.

(23) Cembrero I. (17 de septiembre de 2014). Mas ofrece a Marruecos enseñar 'lengua árabe en horario escolar'. El Mundo, p. 1.

(24) Del Pozo, R. (18 de septiembre de 2014). F-18, no: art. 2 y 155. El Mundo.

(25) Reforma 'sine qua non'. (18 de septiembre de 2014). El País, p. 30.

(26) Bassets, L. (18 de septiembre de 2014). Miércoles de ceniza. El País, p. 2.

(27) Reforma 'sine qua non’. (18 de septiembre de 2014). El País, p. 30.

(28) Garea, F. (18 de septiembre de 2014). Rajoy advierte de los graves riesgos de las consultas de Escocia y Cataluña. El País, p. 11.

(29) Vidal-Folch, X. (20 de septiembre de 2014). Abrumador triunfo de la tercera vía. El País, p. 4.

(30) Vallespín, F. (19 de septiembre de 2014). Se abre el telón. El País, p. 18.

(31) Oppenheimer, W. (19 de septiembre de 2014). El voto que cambió Reino Unido. El País, p. 3.

(32) Tubella, P. (19 de septiembre de 2014). Noche de dedos cruzados en Inglaterra. El País, p. 5.

(33) Barbería, J.L. (20 de septiembre de 2014). Los referendos se cobran su factura. El País, p. 6.

(34) Torreblanca, J.I. (19 de septiembre de 2014). El día después. El País, p. 6.

(35) Gran Bretaña sigue. (18 de septiembre de 2014). La Vanguardia, p. 4.

(36) Escocia vota hoy si rompe con 300 años en el Reino Unido (18 de septiembre de 2014). La Vanguardia, p. 1

(37) Juliana, E. (17 de septiembre de 2014). La culpa es de John Smith. La Vanguardia, p. 5.

(38) González, D. (17 de septiembre de 2014). El fin de los días. La Vanguardia, p. 13.

(39) Domínguez, F. (19 de septiembre de 2014). Flujos soberanistas. La Vanguardia, p. 18.

(40) Puigverd, A. (17 de septiembre de 2014). 'Chicken run'. La Vanguardia, p. 16.

(41) Ónega, F. (18 de septiembre de 2014). Espejo escocés. La Vanguardia, p. 14.

(42) Jorba, R. (20 de septiembre de 2014). Divorcio a la escocesa. La Vanguardia, p. 14.

(43) Ónega, F. (18 de septiembre de 2014). Espejo escocés. La Vanguardia, p. 14.

(44) Vives, X. (18 de septiembre de 2014). Escocia, la libra y la soberanía. La Vanguardia, p. 21

(45) Cuní, J. (18 de septiembre de 2014). Razones y sentimientos. La Vanguardia, p. 6.

(46) Rahola, P. (19 de septiembre de 2014). Una fiestaza. La Vanguardia, p. 25.

(47) Álvaro, F.M. (18 de septiembre de 2014). Escocia, un tabú que cae. La Vanguardia, p. 24.

(48) Íbidem.

(49) Ponti, F. (17 de septiembre de 2014). 'Win-win'. La Vanguardia, p. 23.

(50) Rahola, P. (18 de septiembre de 2014). Buenos días, Escocia. La Vanguardia, p. 21. 
* Esta investigación se ha realizado dentro de las actividades del Grupo de Investigación en Historia Reciente (GIRHE)", financiado por la Universidad de Navarra.

\section{BREVE SEMBLANZA DE LAS AUTORAS}

Itziar Reguero Sanz es Licenciada en Periodismo y Premio Extraordinario en el Máster en Investigación de la Comunicación como Agente Histórico Social (UVa). Desde 2014, Reguero es Investigadora Predoctoral de la Universidad de Valladolid, con una beca de Formación del Profesorado Universitario (MECD). Sus líneas de investigación se centran en el papel de los mass media en la formación del Estado Autonómico y en las relaciones de poder que se establecen entre políticos y medios, así como su posterior influencia en la sociedad.

Marta Requejo Fraile es Licenciada en Periodismo y Máster en Investigación de la Comunicación como Agente Histórico Social por la Universidad de Valladolid (UVa). Desde 2014, es Investigadora Predoctoral de la UVa con una beca de Formación del Profesorado Universitario del Ministerio de Educación Cultura y Deporte. Su principal línea de investigación es la comunicación en procesos de paz y conflictos desde una perspectiva deontológica y ética.

Ana María Velasco es periodista e historiadora del arte y actualmente es investigadora predoctoral de la Universidad de Valladolid. Ha realizado el máster de Investigación de la Comunicación como Agente Histórico Social y el curso de posgrado en Historia y Estética de la Cinematografía de la UVa. Su tesis investiga sobre la mujer, la moda y el cambio social en el paso del franquismo a la democracia. Es miembro del Grupo de Innovación Docente "Enseñanza en Igualdad e Inclusión de Género".

Ámbitos. Revista Internacional de Comunicación, n.29, año 2015, tercer trimestre (verano). Recibido: 01/04/2015

Aprobado: 12/06/2015 